\title{
THE ROLE OF OXIDATIVE STRESS IN THE PATHOGENESIS OF VASCULAR COMPLICATIONS IN CHILDREN WITH INSULINABLE SUGAR DIABETES
}

\section{A.A. Basov' , L.G. Ivchenko', D.A. Domenyuk', T.D. Dmitrienko ${ }^{3}$, C.V. Nuzhnaya ${ }^{4}$}

\author{
${ }^{1}$ Department of Fundamental and Clinical Biochemistry, Kuban State \\ Medical University of the Ministry of Healthcare of the Russian Federation, \\ Sedina Street, 4, Krasnodar, Russia, 350063.E-mail: ilyaMB@ksma.ru, \\ tel: +78612680230 \\ 2 Department of General Practice Dentistry and Child Dentistry, Stavropol \\ State Medical University of the Ministry of Healthcare of the Russian \\ Federation, 310, Mira Street, Stavropol, Russia 355017. \\ E-mail:domenyukda@mail.ru, tel:+79188701205. \\ ${ }^{3}$ Department of Dentistry, Pyatigorsk Medical-Pharmaceutical Institute \\ (Branch of Volgograd State Medical University, Ministry of Healthcare, \\ Russian Federation), 11, pr. Kalinina, Pyatigorsk-32, Stavropol Region, \\ Russia357532.E-mail:s.v.dmitrienko@pmedpharm.ru, \\ tel: +78793324474. \\ ${ }^{4}$ North Caucasus Federal University, Institute of living systems, Russian \\ Federation, Stavropol, Pushkin Street, 1, building 3, Russia 355000. \\ E-mail:knuz97@mail.ru, tel: +79197305161.
}

ABSTRACT - The focus of this article is to evaluate lipid peroxidation, antioxidant protection and oxidative stress indicators based on the neutrophil granulocytes functional activity model in children with Type 1 Diabetes Mellitus (T1D). The studies have revealed that, in case of negative effects of reactive oxygen species, children with T1D had intensification in the lipoperoxidation and activation of the antioxidant defense system, including bidirectional changes in non-enzymatic mechanisms. Coordinated generation of reactive oxygen species and oxygen-dependent blood cell metabolism in children with compensated T1D is indicative of the oxidative stress second stage (resistance) development. Reduced production of reactive oxygen species, decrease in the phagocyte oxygendependent metabolism activation, incomplete phagocytosis mechanisms correlated with an increase in the pancreas affected area (destruction of insulin-producing $\beta$-cells) in children with decompensated T1D, points at the onset of the third stage (depletion) of oxidative stress. Metabolic disorders in children with T1D are determined by the intensity of the "respiratory explosion" of neutrophilic granulocytes in the Lipid peroxidation and antioxidant protection system.

KEYWORDS - type 1 diabetes mellitus, child population, chemiluminescence, neutrophil granulocytes, lipid peroxidation, antioxidant protection.

\section{Article history:}

Received 4 March 2019

Received in revised form 20 March 2019 Accepted 20 March 2019

\section{INTRODUCTION}

Latest data indicates that prevalence of diabetes mellitus (DM) in the world increased during the last decade more than two-fold, reaching some 415 million patients by the end of 2015 . According to the current estimation by the International Diabetes Federation, 642 million patients will be suffering from DM by 2040. These observations resulted in the UN Resolution on Diabetes 61/225 passed on 20.12.2006, and in 2011 - UN Political Declaration, addressed to national health systems, calling for the establishment of multidisciplinary strategy in the prevention and control of non-communicable diseases, where special attention is drawn to the problem of diabetes as one of the leading causes of disability and mortality $[1,-9]$.

Like many other countries, Russian Federation experiences a sharp rise in the prevalence of DM. According to Russian Federal Diabetes Register, there are at least 4.35 million patients with DM in this country by the end of 2016 (3\% of population) with $92 \%$ ( 4 million) - Type 2 DM, 6\% (255 th) - Type 1 DM and $2 \%(75 \mathrm{th})-$ other types of DM. However, these results underestimate real quantity of patients, because they consider only registered cases. Results of Russian epidemiological study (NATION) confirmed that only $50 \%$ of Type $2 \mathrm{DM}$ are diagnosed. So real prevalence of patients with DM in Russia is no less than 8-9 million patients (about 6\% of population). This is a great long-term problem, because a lot of patients are not diagnosed, so they don't receive any treatment ant have high risk of vascular complications [10-17].

According to the National register, 22,969 children and 8,758 adolescents with T1D had been identified in Russia by 2017. The prevalence of T1D as per 100,000 persons was 86.73 for the child category, and 203.29 for the category of adolescents, while the morbidity and mortality indices were 11.78 and 8.03 , respectively and 0.05 and 0.10 , respectively [18-20].

Severe consequences of the global pandemics of DM include its vascular complications: nephropathy, retinopathy, coronary, cerebral, coronary and peripheral vascular disease. These conditions are responsible forth majority of cases of diabetes-related disability and death [21-23]. 
Lipoperoxidative mechanisms play a significant role in the human body life activity. There is research data available proving that free radical reactions occurring at a low level of functional activity are universal modifiers for the structure and function of cell membranes, having a direct involvement in their recovery (renewal). There is evidence to prove a role played by free-radical oxidation reactions in microbial lysis and phagocytosis. The role of lipoperoxidative mechanisms in cell division and nerve impulse conduction has been established, too [24-27].

Free radicals are atoms, structural fragments of molecules or whole molecules, which have unpaired electrons in the external orbitals. Free radicals have an extremely high reactivity thus interacting with various molecules, causing their damage. Potentiation of lipid peroxidation contributes to a sharp (abrupt) increase in the level of free radical derivatives and exo-, endocellular superoxidant oxygen metabolites that have a direct toxic effect. A high level of functional activity in free-radical and peroxide reactions has been observed in case of many external (industrial pollution; hyperbaric oxygenation; hypoxia; vibration; exposure to electromagnetic fields, radioactive and ultraviolet radiation) and internal (low levels of antioxidants; stress; effect of synthetic drugs and xenobiotics; hypodynamia; aging; excessive consumption of fats and carbohydrates) factors affecting the human body. Peroxidation syndrome plays a significant role in the pathogenesis of malignant neoplasms, communicable diseases, atherosclerosis, heart attack, stroke, coronary heart disease, peptic ulcer and burn disease, bronchopulmonary pathology, adaptive overstrain syndrome, diabetes [28-32].

The results obtained by Russian and foreign researchers indicate that oxidative stress and activation of lipid peroxidation are the proven pathogenetic mechanisms of T1D. A significant increase of blood plasma glucose levels through glycation, glucose auto-oxidation, as well as polyol pathway intracellular activation, which potentiates the $\mathrm{NADH} / \mathrm{NAD}+$ ratio imbalance, contributes to free radicals excessive development and accumulation. The metabolic changes (hyperglycemia, dyslipidemia, insulin secretion change, reduced antioxidant reserve) observed in case of T1D trigger mechanisms activating the functional status of cell membranes, as well as mechanisms that activate lipid inflammation mediators, which control lipid peroxidation and antioxidant protection in the damaged area. The key condition for lipoperoxidation is the development of reactive oxygen species. Given that, an objective assessment of the oxidative stress intensity in T1D implies investigating the severity of the homeostatic imbalance, which is due to neu- trophils oxygen consumption with the development of oxygen-dependent bactericidal activity that is required to eliminate the agents [33-40].

Systematizing the published research data, we can state that identifying the patterns of free-radical processes and the specific features within the functioning of various antioxidant protection components, as well as oxygen-dependent neutrophils metabolism in children with T1D, will allow specifying early diagnostic criteria for endocrine pathologies, also adding to scientific understanding of the change pattern in lipoperoxidation intensity at different stages of the disease compensation. Besides, the outcomes will increase the explanatory value of diagnostic and prognostic criteria in pediatric practice, also validating the approach of viewing the body as a whole integrated system, and thus contributing to a search for integrated solutions in making treatment and rehabilitation arrangements for endocrine diseases.

Aim of study

To assess the status of lipid peroxidation, antioxidant protection and oxidative stress indicators based on the functional activity model of neutrophilic blood granulocytes in children with T1D depending on the endocrinopathy compensation stage.

\section{MATERIALS AND METHODS}

Prior to carrying out the research involving children, a conclusion was obtained from the Committee on Bioethics as well as expressed voluntary consent from the parents (custodians). The results of the ethical review confirmed the research protocols compliance with national and international regulations - the World Medical Association Declaration of Helsinki, 1964 ETHICAL PRINCIPLES FOR MEDICAL RESEARCH INVOLVING HUMAN SUBJECTS, as amended by the WMA LXIV General Assembly (2013); Cl. 24 of the Russian Federation Constitution; Rules of Clinical Practice in the Russian Federation (Decree 266 issued by the Ministry of Healthcare of the Russian Federation on June 19, 2003); ethical standards by the Committee on Experiments, Standards for Clinical Trials (GOST R 52379-2005); Federal Law of the Russian Federation 323-FL ON THE PRINCIPLES OF THE PROTECTION OF CITIZENS' HEALTH IN THE RUSSIAN FEDERATION (of 11/21/2011).

Clinical and laboratory diagnostic studies involving 121 children in their second childhood period (boys - aged 8-12; girls - 8-11) were carried out after obtaining voluntary consent from the parents (guardians). All the patients were divided into two groups. The comparison group included 32 children 
(Health Groups I and II; Veltischev, 1994). The diagnosis of healthy was given subject to the Pediatrician's conclusion. The main group (89 people) included children diagnosed with T1D who were undergoing treatment in the Endocrinology Departments, G. K. Filippsky Child Clinical Hospital (City of Stavropol, Russia) and Child Regional Clinical Hospital of Krasnodar (Russia) within the period of 2010-2017. The patients of the main group, depending on the endocrinopathy compensation degree, were divided into two subgroups - Subgroup 1 included 46 children (52.9\%) with compensated T1D, while Subgroup 2 included 43 children (47.1\%) with decompensated T1D. According to the disease history of the children with T1D, 27 patients (30.3\%) had had the disease for up to 1 year; 43 patients $(48.3 \%)-1$ to 5 years, whereas in another 19 patients $(21.4 \%)$ the disease duration exceeded 5 years.

In the category with the endocrinopathy duration up to one year, dominating were children with decompensated T1D (20 persons, $74.1 \%$ ), whereas compensated T1D was observed in 7 children only (25.9\%). The division of the T1D-diagnosed child population endocrinopathy based on the compensation degree followed the criteria of carbohydrate metabolism compensation (Dedov I. I., 2007). The glycemia level indicators were recorded subject to the child's clinical history.

The T1D diagnosis in the observed groups was made according to the laboratory test outcomes (general blood test; urine test; biochemical blood test with blood glucose level assessment), as well as following clinical examination data obtained from the Endocrinologist in a hospital.

The material used for the study of antioxidant defense indicators and lipid peroxidation was blood serum and hemolysate prepared from erythrocytes. Blood sampling from the ulnar vein was performed using a vacuum system (venipuncture), which was carried following the generally established algorithm for blood sampling from a vein, taken in the morning and on an empty stomach. The antioxidant protection status was evaluated through the following parameters:

- Total antioxidant activity (AOA). For this, a model system was used, which included a suspension of chicken egg yolk lipoproteins, that allowing studying the blood serum capacity to slow down the accumulation of active products by thiobarbituric acid (TBA) in the suspension (G.I. Klebanova, 1988).

- Superoxide dismutase activity (SOD). The SOD activity was evaluated on a spectrofluorophotometer $(\lambda=320 \mathrm{~nm})$ using a curve that reflected the enzymatic inhibition of adrenaline auto-oxidation. The unit of enzymatic activity was taken as the amount of SOD required for a $50 \%$ inhibition of adrenaline auto-oxidation into adrenochrome (H.P. Misra, I. Fridovich, 1972).

- Retinol and $\alpha$-tocopherol were identified via the fluorometric method (R.Ch. Chernyauskene, 1984). All-trans-retinol (Sigma) and L,D, $\alpha$-tocopherol (Serva) were used as the external standard.

- The content of reduced (GSH) and oxidized (GSSG) glutathione was identified through the fluorometric method under the same conditions for fluorescence recording (P.J. Hissin and R. Hilf(1976)). The measurements were taken on a spectrofluorophotometer at $\lambda=350 \mathrm{~nm}$ and $\lambda=420 \mathrm{~nm}$, respectively.

Lipid peroxidation intensity was studied by the level of substrates with conjugated double bonds (DB). Diene conjugates (DC), ketodienes (KD) and conjugated trienes (CT) were identified using a spectrophotometric method based on measuring, in the optical region, the absorbance of electromagnetic radiation of conjugated lipid hydroperoxides' diene structures: $\mathrm{DB}(\lambda=220 \mathrm{~nm}), \mathrm{DC}(\lambda=232 \mathrm{~nm}), \mathrm{KD}$ and CT $(\lambda=278 \mathrm{~nm})$ (J. Stocks method (1974), modification by I. Volchegorsky $(1989,2000))$. The principle of the method for studying malondialdehyde (MDA), which is the end product of free-radical polyunsaturated higher fatty acids oxidation, is based on irreversible protein denaturation that occurs in case $\mathrm{NH} 2$ protein groups interact with MDA aldehyde groups. The reaction of MDA with TBA produced a trimethyl complex (stained compound). The optical density of TBA-active lipoperoxidation products was assessed fluorimetrically $(\lambda=532 \mathrm{~nm})$, and the MDA concentration was calculated based on the molar extinction coefficient of the trimethyl (stained) complex (V.B. Gavrilova, (1987)).

The total lipids level (TL) was studied with the spectrophotometric method using a semi-automatic biochemical analyzer BioChem SA (High Technology Inc., USA) using a reagent kit (Spinreact, Spain). Measurements were taken on an RF-5301PC spectrofluorophotometer (SHIMADZU, Japan) and a CM2203 spectrofluorometer (Solar, Belarus).

The oxidative stress coefficient (OSC) was calculated in order to reach an objective assessment of the antioxidant protection and lipid peroxidation. This coefficient is a ratio of the antioxidant protection and lipid peroxidation values in children with compensated T1D (Subgroup 1) and decompensated T1D (Subgroup 2) to the average values in healthy children (comparison group). When the OSC value exceeded 1 , the oxidative stress was registered.

For a deeper in vivo study of the free-radical oxidation status in the studied groups, the functional activity of neutrophilic granulocytes was investigated employing the chemiluminescent (CL) method (De 
Sole P., (1983)). The principle of the chemoluminescent analysis method is based on the registration of a quantum flux that emerges when a substance passes from an electronically excited state to the ground state. The assessment of luminol-dependent spontaneous chemiluminescence (LDCL) and zimosan-induced chemiluminescence (ZICL) was carried out for 90 minutes on a 36-channel analyzer CL3604 (Russia). The analyzer fluorescence intensity of $5.1 \times 105$ quanta per second was taken as $1 \mathrm{cu}$ (Fig. 1).

The following indicators laid the basis for the

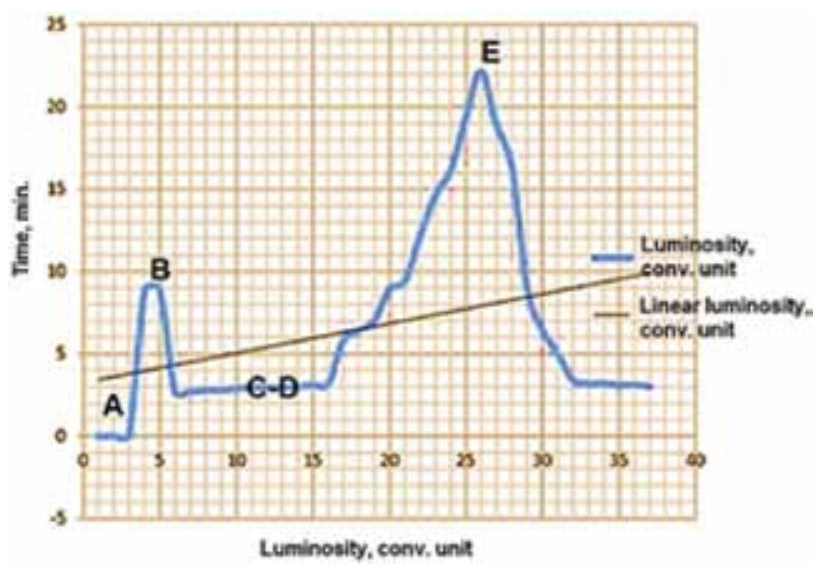

Fig. 1. Vein blood spontaneous $L D C L$ curve: $A$ - spontaneous fluorescence; $B$ - fast flash; $C, D$ - latent period; $E$ - slow fluorescence phase

outcomes: Tmax - time to reach the maximum; Imax — the maximum intensity level; $S$ - the area under the CL curve; the activation index $(\mathrm{AI})$ - the ratio of the ZICL area to the area of LDCL, which determines the CL enhancement induced by zymosan. The analyzer control along with the result record was performed through a PC. Statistical processing, including the data systematization as well as the graphic images and tables construction was carried out following variation statistics methods. The results can be seen as the arithmetic mean and its standard error. The differences significance between the groups $(\mathrm{p})$ was assessed subject to Student's t-test. The differences in indicators were considered significant at $\mathrm{p}<0.05$. The calculations were performed employing the following software: STATISTICA 10.0, DBASE, STATGRAF, STAT4 (Stat Soft Inc., USA), as well as Med Calc (version 9.3.5.0), SPSS (version 7.5).

\section{RESULTS AND DISCUSSION}

The antioxidant defense system includes indirect and direct components. The indirect component im- proves the basic metabolism, which does not involve the generation of excessive amounts of lipid peroxidation products and reactive oxygen species. Following this, an optimal way to regulate the oxygen-peroxide status and the dependent signaling pathways determining the course of all fundamental cellular processes is to change the mitochondrial base, which includes the activity, as well as quantitative and qualitative mitochondria components. The direct component of antioxidant defense includes a set of low- and macromolecular compounds of endogenous origin. A key role in the antioxidant defense system is played by antioxidant enzymes - the first defense line factors against free-radical oxidation and lipoperoxidation products, which include the enzymes complex of the glutathione system (GR, GPO, GST), peroxidase, catalase, myeloperoxidase, paraoxonase, aconitase, and superoxide dismutase.

Table 1 shows the indicators of antioxidant defense in the patients of the said groups.

Under physiological conditions, the human body reveals a balanced equilibrium between the level of oxidants (free radicals) and the antioxidant defense activity status. Oxidative stress provokes an increase in the number of free radicals that work a damaging effect on cellular structures. An increase (accumulation) of free radicals in body tissues disturbs the system balance. One of the significant parameters regulating the antioxidant defense system's buffer capacity is the total AOA, which includes a large number of nonenzymatic and enzymatic links. Of the basic enzymatic components, mention is to be made of fat-soluble vitamins (retinol, $\alpha$-tocopherol) and superoxide dismutase. There is research-based evidence showing that $\alpha$-tocopherol, which is an essential component of all plasma membranes, promotes the development of low-active radicals that are not capable of maintaining lipid peroxidation chain reactions. Besides, this fatsoluble vitamin makes membrane phospholipids less accessible for peroxidation by increasing their packing density. Children diagnosed with T1D, if compared with healthy ones, reveal activation of the antioxidant defense system (Table 2). In our opinion, this condition in the main group children, which demonstrated an increase in the total AOA (compensation stage $11.7 \pm 0.7 \%$; decompensation stage $-40.4 \pm 2.3 \%)$, a decrease in the retinol concentration $(5.6 \pm 0.3 \%$ and $25.7 \pm 1.4 \%$, respectively) and $\alpha$-tocopherol $(6.1 \pm 0.2 \%$ and $32.6 \pm 1.7 \%$, respectively), is to be considered a protective mechanism in response to an increasing generation of reactive oxygen species (oxidative stress) aimed to reduce the severity of the endocrinopathology. Glutathione has been proven to perform its protective properties in its reduced (GSH) form only, while 
Table 1. Indicators of antioxidant protection in patients of the studied groups, $(M \pm m),(p \leq 0,05)$

\begin{tabular}{l|l|l|l}
\hline \multirow{2}{*}{$\begin{array}{l}\text { Indicators, } \\
\text { units of measurements }\end{array}$} & Research groups & \multicolumn{2}{l}{} \\
\cline { 2 - 4 } & Comparison group & First group & The second group \\
\hline Total antioxidant activity, c.u. & $15,07 \pm 1,61$ & $16,84 \pm 1,19$ & $21,16 \pm 1,33$ \\
\hline Retinol, mkmol / & $1,87 \pm 0,19$ & $1,76 \pm 0,16$ & $1,39 \pm 0,28$ \\
\hline a-tocopherol, mkmol / & $6,69 \pm 0,58$ & $6,28 \pm 0,43$ & $4,51 \pm 0,67$ \\
\hline Recycled glutathione, mkmol / & $2,72 \pm 0,13$ & $2,66 \pm 0,11$ & $2,57 \pm 0,16$ \\
\hline Oxidized glutathione, mkmol / & $1,78 \pm 0,19$ & $1,84 \pm 0,22$ & $2,17 \pm 0,14$ \\
\hline Superoxide dismutase, c.u. & $1,52 \pm 0,08$ & $1,46 \pm 0,06$ & $1,27 \pm 0,11$
\end{tabular}

Table 2. Indicators of lipoperoxidation in patients of the studied groups, $(M \pm m),(p \leq 0,05)$

\begin{tabular}{l|l|l|l}
\hline \multirow{2}{*}{$\begin{array}{l}\text { Indicators, } \\
\text { units of measurements }\end{array}$} & Research groups & \multicolumn{2}{l}{} \\
\cline { 2 - 4 } Comparison group & First group & The second group \\
\hline Conjugated double bond substrates, c.u. & $1,41 \pm 0,14$ & $1,53 \pm 0,11$ & $1,92 \pm 0,19$ \\
\hline Diene conjugates, mkmol / & $0,48 \pm 0,04$ & $0,57 \pm 0,03$ & $0,86 \pm 0,09$ \\
\hline Ketodienes and conjugated trienes, c.u. & $0,16 \pm 0,02$ & $0,19 \pm 0,03$ & $0,26 \pm 0,05$ \\
\hline Malonic dialdehyde, mkmol / & $1,46 \pm 0,13$ & $1,59 \pm 0,12$ & $2,29 \pm 0,17$ \\
\hline Total lipids, g/I & $4,09 \pm 0,27$ & $4,37 \pm 0,38$ & $5,96 \pm 0,59$
\end{tabular}

shifts in the glutathione status have a negative effect on complications development and the disease outcome. Enhanced glutathione development in its oxidized (GSSG) form (3.4 $\pm 0.2 \%$ and $21.9 \pm 1.3 \%$, respectively) against a decrease in the SOD activity $(3.9 \pm 0.4 \%$ and $16.4 \pm 0.9 \%$, respectively) and the glutamine content in the reduced (GSH) form $(2.2 \pm 0.2 \%$ and $5.5 \pm 0.3 \%$, respectively) in children with $\mathrm{T} 1 \mathrm{D}$, compared with healthy children's indicators, points not only at the activation of the antioxidant defense system and the stress in the the glutathione redox system, yet also is indicative of a slowing lipid chain oxidation, since the SOD action is aimed primarily at superoxide radicals removal. The study of the glutathione system in erythrocytes in children with T1D indicates a decrease in the activity of GSH - the main component of the antioxidant system, which, from our point of view, is a result of the damaging effect wrought by reactive oxygen species. Lower level of GSH increases the accessibility of membranes for the toxic action of lipid peroxidation products. A decrease in the cells antioxidant defense, which manifests itself in an increased GSSG concentration, accelerates the inactivation and oxidation of the thiol protein groups, thus aggravating the oxidative stress.

It has been scientifically proven that insulin deficiency (absolute, relative) in T1D contributes to an increase in the lipid peroxides concentration. The effect of the insulin hormone, which acts as a lipid peroxida- tion inhibitor, is not only aimed at utilizing peroxide compounds and enhancing the membrane lipids mobility (lability), but also includes bidirectional changes in the non-enzymatic part (protein glycosylation). Along with an increase in the endocrinopathy severity, there is a progress in the lipid peroxidation activity, which is manifested through a cytotoxic effect. The development of this pathophysiological process that implies inactivation (inhibition) of membrane-bound enzymes (cytochrome oxidase activity), is seen as damage to erythrocyte membranes and lysosomes. Emerging morphological, structural and functional changes in endothelial cells and smooth muscle elements of the vascular wall, which often lead to rupture, facilitate vascular complications and diabetic angiopathies.

Table 2 shows the lipoperoxidation indicators in the patients of the involved groups.

Children with T1D, compared with healthy children, have all the respective indicators showing lipid peroxidation intensification: an increase in the substrates with conjugated double bonds (compensation stage, $8.5 \pm 0.5 \%$; decompensation stage, $36.2 \pm 2.1 \%$ ); DC accumulation (lipid peroxidation primary products) $(18.8 \pm 1.2 \%$ and $79.2 \pm 3.7 \%$, respectively); accumulation of KD and CT (the intermediate products of lipoperoxidation) $(18.7 \pm 1.4 \%$ and $62.5 \pm 3.1 \%$, respectively); an increase in the MDA level (the final product of lipid peroxidation) $(8.9 \pm 0.7 \%$ and $56.8 \pm 2.7 \%$, respectively); total lipids $-6.8 \pm 0.4 \%$ 
and $45.7 \pm 2.3 \%$, respectively. Note to be made that children with decompensated T1D have a significant accumulation of malonic dialdehyde, the most toxic product. From our point of view, in children with decompensated T1D, the most prominent increase in the lipid peroxidation initiation at the stage of primary, intermediate and final products - in comparison with the antioxidant defense system activation parameters - contribute to thickening of the blood vessel walls basement membrane, a higher blood viscosity, and a slower blood flow thus increasing the probability of intravascular coagulopathy (aggregation of blood cells) and disturbance through various hemostasis stages.

Table 3 shows the range of oxidative stress coefficient fluctuations in the studied groups.

Table 3. The range of fluctuation of the coefficient of oxidative stress in patients of the studied groups, (c.u.), $(M \pm m),(p \leq 0,05)$

\begin{tabular}{l|l|l|}
\hline Research groups \\
\hline Comparison group & First group & The second group \\
\hline Less than 1 & $1,03-1,18$ & $1,08-1,79$ \\
\hline
\end{tabular}

Antioxidant defense is a complex multilevel system that blocks the transition of lipid peroxidation processes from a physiological into a pathological status (oxidative stress). Oxidative stress of varying severity, developing due to disturbed antioxidant defense mechanisms, not only accompanies the course of classical stress, yet can also manifest itself as a key factor in the pathological condition. In children with long-term decompensated T1D, the probability of oxidative stress occurrence (OSC above 1) increases significantly due to disturbed components interaction (redox metabolism and vascular complications) in a single stress mechanism of all physiological systems.

An increase in the free-radical oxidation activity, regarded as a reliable indicator of the macroorganism status comes along with morphological and functional impairments in biological membranes. The set of these processes, which include a reduction in the membranes lipid layer stability, enhanced peroxidation of proteins, lipids, and ion permeability, serve the basis for the pathogenesis of various diseases at the molecular level. The use of CL analysis, which is highly informative, sensitive, and reliable, allows objective and reliable identification of potential morphological and metabolic disorders in the development and progress of the endocrine pathology at the molecular level (studying photochemical reactions, electronically excited molecule states, structure and properties of biological systems, and molecular transitions dynamics).
According to the available data obtained through investigating biological structures using CL analysis, there has been their connection identified with freeradical oxidation in a macroorganism, which is due to molecular oxygen reduction to active species (hydroxyl and superoxide anion radicals, singlet oxygen) has been established. The most important source of reactive oxygen species is lipid auto-oxidation, which occurs via release of free radicals. In case of peroxides recombination developing through interaction of free radicals with oxygen, there occurs emission of light quanta. The emission of photons is also observed upon excitation of ketones, oxygen dimer molecules, aldehydes, oxalates, cyclic hydroperoxides, aldehydes, biogenic amines, decomposition of intermediate products of reaction with molecular oxygen (peroxides). Inhibition of free-radical oxidation in the body goes on through natural antioxidants of hydrophilic (ascorbic acid, sulfhydryl compounds of the SH-group of proteins) and hydrophobic (flavins, tocopherols, steroids, carotenoids) phases.

Given this point of view, CL activity points at not excessive free-radical oxidation only, yet also reveals a low activity (lack) of antioxidants. Investigating the spontaneous emission intensity proportional to the free radicals recombination rate, does not offer a reliable picture of the reasons behind the change in the free-radical oxidation rate. In this connection, notable is the method of modifying free-radical reactions using luminol with a subsequent analysis of induced CL. In the presence of reactive oxygen species, luminol is oxidized to develop electron-excited carbonyl chromophores. The identified functional groups with a high quantum yield increase significantly the glow intensity due to the development of reactive oxygen species. This phenomenon is used successfully to study the functional level of phagocytic immunity. Insufficient generation of reactive oxygen species, aimed at antigens inactivation, means reduced activation rate of oxygen-dependent phagocytic metabolism, as well as incomplete phagocytosis.

There is scientifically proven data showing that the mechanisms of non-specific immunity are the initial stages where antigens (foreign agents) come into contact with the body. Neutrophil granulocytes that have a high reactivity, in response to numerous signals concerning disturbed internal environment can perform rapid functional restructuring, thus determining the nature of the inflammation course. The so-called respiratory (oxygen) explosion, which is caused by a sharp rise in the oxygen use through its conversion by phagocytes into active species, determines the neutrophils mobilization rate, potentiating the launch of the body's defense systems. The ability of neutrophilic 
blood granulocytes to develop a sufficient number of reactive oxygen species is a prognostic sign of the nature (type) of inflammation, whereas the response to stimulation allows objective evaluation of the body defense activity. The model of neutrophilic granulocytes with high diagnostic significance used to study oxidative stress allows a significant expansion of the information pool to be obtained from the assessment of free-radical oxidation, which is viewed as a factor that indicates the health status through different phases of T1D in children.

Table 4 shows the indicators of luminol-dependent chemiluminescence of neutrophilic blood granulocytes in patients within the above-mentioned groups.
In case of an increasing severity of endocrinopathy in children with T1D, if compared with the main group (Subgroup1), there were also bidirectional changes registered in the spontaneous LDCL values (a 1.9 decrease in Imax, and a 1.7 times decrease in $S$; Tmax parameters growth - by 1.5 times) and zymosan-induced LDCL (Imax decrease -1.5 times, $S-1.4$ times; Tmax parameters growth -1.6 times). The reduction of nonspecific antimicrobial defense in children with decompensated T1D, occurring along with an increasing pancreas lesion area (destruction of insulin-producing $\beta$-cells of the Langerhans islets), is an effect of the following pathophysiological mechanisms:

Table 4. Indicators of luminol-dependent chemiluminescence of neutrophilic blood granulocytes in patients of the studied groups, (M $\pm m)$

\begin{tabular}{|c|c|c|c|c|c|c|}
\hline \multirow{3}{*}{ Indicators } & \multicolumn{6}{|c|}{ Research groups } \\
\hline & \multicolumn{2}{|c|}{ Comparison group } & \multicolumn{2}{|l|}{ First group } & \multicolumn{2}{|c|}{ The second group } \\
\hline & Value range & Average value & Value range & Average value & Value range & Average value \\
\hline \multicolumn{7}{|c|}{ Spontaneous chemiluminescence } \\
\hline Tmax., s & $573,4-1478,7$ & $905,3 \pm 34,1$ & $417,6-1084,9^{*}$ & $667,3 \pm 23,8^{*}$ & $614,6-1583,5^{*}$ & $968,9 \pm 31,7^{*}$ \\
\hline $\operatorname{Imax}$, o.e. $\times 10^{3}$ & $3,03-12,96$ & $9,93 \pm 0,67$ & $28,87-80,33^{* *}$ & $51,46 \pm 3,78^{* *}$ & $16,23-43,91^{* *}$ & $27,68 \pm 2,14^{* *}$ \\
\hline S, $0 . e . \times 10^{5}$ & $2,82-6,74$ & $3,92 \pm 0,31$ & $17,58-49,22^{* *}$ & $31,64 \pm 2,06^{* *}$ & $9,84-28,21^{* *}$ & $18,37 \pm 1,95^{* *}$ \\
\hline \multicolumn{7}{|c|}{ Zymosan-induced chemiluminescence } \\
\hline Tmax., s & $772,8-1297,4$ & $1035,1 \pm 38,6$ & $593,9-1311,3^{*}$ & $717,2 \pm 26,3^{*}$ & $472,2-1559,2^{*}$ & $1087,0 \pm 43,1^{*}$ \\
\hline Imax, o.e. $\times 10^{3}$ & $9,72-29,06$ & $19,34 \pm 1,26$ & $36,63-135,16^{* *}$ & $98,53 \pm 5,34^{* *}$ & $34,18-99,57^{* *}$ & $65,39 \pm 4,02^{* *}$ \\
\hline S, o.e. $\times 10^{6}$ & $3,19-10,32$ & $7,13 \pm 0,58$ & $30,46-82,03^{* *}$ & $51,57 \pm 3,29^{* *}$ & $16,44-51,16^{* *}$ & $34,72 \pm 2,19^{* * *}$ \\
\hline Activation index & & 1,82 & & 1,63 & & 1,89 \\
\hline
\end{tabular}

Note: statistically significant differences with the children of the comparison group $\left({ }^{*}-p<0.05 ; *^{* *}-p<0.01\right)$.

The results of studying the luminol-dependent chemiluminescence parameters in neutrophilic blood granulocytes in the main group indicated that children with T1D in the compensation stage have bidirectional change dynamics in the spontaneous LDCL if compared with similar factors in healthy children (an increase of Imax - 5.2 times and S - 8.1 times; a decrease in Tmax values 1.4 times). This type of change in the values indicates a proper generation of reactive oxygen species and blood cells oxygen-dependent metabolism processes in response to the early phases of selective organ-specific destruction of insulinproducing $\beta$-cells in the Langerhans pancreatic islets. A decrease in the maximum intensity growth rate value (Imax) during zymosan-induced LDCL in this subgroup, in comparison with the change dynamics of spontaneous LDCL, confirms that the development of the destruction initial stage in the islet cells correlates with a decrease in the reserve capacity of neutrophil granulocytes antimicrobial protection.
- reduced rate of metabolic processes, which is accompanied with a respiratory burst developing;

- reduced production of reactive oxygen species;

- depleted phagocytic activity of macrophages.

From our point of view, this condition points at a long chronic inflammatory process, which is combined with the depletion of defense $\&$ compensation mechanisms aimed at improving the body's vital processes under inflammation.

\section{CONCLUSIONS}

1. In children with T1D, through all the phases of the disease, the antioxidant defense system is activated against the negative effects of reactive oxygen species, including bidirectional changes in non-enzymatic mechanisms. The increase in the overall antioxidant activity (compensation stage, $11.7 \pm 0.7 \%$; decompensation stage, $40.4 \pm 2.3 \%$ ), a decrease in the retinol level $(5.6 \pm 0.3 \%$ and $25.7 \pm 1.4 \%)$ and $\alpha$-tocopherol $(6.1 \pm 0.2 \%$ and 
$32.6 \pm 1.7 \%)$, compared with healthy children, is a protective response to the increasing generation of reactive oxygen species, the aim of that being reduction of the endocrinopathy severity course

2. A significant increase in the content of oxidized glutathione (GSSG) (compensation stage, $3.4 \pm 0.2 \%$; decompensation stage, $21.9 \pm 1.3 \%$ ) in the venous blood of children with T1D, compared with healthy children, against a decrease in the superoxide dismutase activity $(3.9 \pm 0.4 \%$ and $16.4 \pm 0.9 \%)$ and the level of reduced glutathione $(\mathrm{GSH})(2.2 \pm 0.2 \%$ and $5.5 \pm 0.3 \%)$, is indicative of a stress in the glutathione redox system, as well as a slow chain oxidation of lipids. The redox system stress results in inhibited anti-peroxide enzymes and a decrease in the antioxidants level, which contribute to the stability of the antiperoxide and antiradical cell potential.

3. Children with T1D, reveal intensified lipid peroxidation through all the stages of the pathology. Compared with healthy children, an increase is registered in the level of substrates with conjugated double bonds (compensation stage, $8.5 \pm 0.5 \%$; decompensation stage, $36.2 \pm 2.1 \%$ ), accumulation of diene conjugates $(18.8 \pm 1.2 \%$ and $79.2 \pm 3.7 \%)$, accumulation of ketodienes and conjugate trienes $(18.7 \pm 1.4 \%$ and $62.5 \pm 3.1 \%)$, as well as an increase in the malondialdehyde level $(8.9 \pm 0.7 \%$ and $56.8 \pm 2.7 \%)$ and in total lipids, $6.8 \pm 0.4 \%$ and $45.7 \pm 2.3 \%$, respectively.

4. In children with decompensated T1D, prominent lipid metabolism disturbances combined with oxidative stress indicators, aggravate the course of the endocrine pathology, increasing significantly the likelihood of intravascular complications (coagulopathy).

5. Highly reliable and diagnostically significant risk factors for early development of microvascular angiopathies should include the level of total antioxidant activity, as well as the content of diene conjugates and total lipids in the venous blood.

6. The analysis of the outcomes obtained through the study of neutrophilic blood granulocytes functional activity in children with T1D, using luminol-dependent chemiluminescence indicates an increase in the intensity of reactive oxygen species development not only during a spontaneous chemiluminescent reaction, yet also zymosan-induced (stressed) chemiluminescence, if compared with healthy children.

7. The method of spontaneous and induced luminol-dependent chemiluminescence employed to study oxidative stress using the model of neutrophilic granulocytes in children with T1D is a reliable, cost-effective, highly sensitive express method for assessing the phagocytic immunity functional status, which, at the same time, allows registering the kinetic component of the phagocytosis process.

8. Children with decompensated T1D have changes in the Lipid peroxidation and antioxidant defense system, which is due to intensified lipid peroxidation against activated antioxidant defense mechanisms, and such changes correspond to the systemic inflammatory response syndrome that occurs with the maximum stress in the body's protective-compensatory mechanisms.

\section{REFERENCES}

1. International Diabetes Federation. Diabetes Atlas $7^{\text {th }}$ Edition. 2015.

2. Dedov I.I., Shestakova M.V., Vikulova O.K. Standards of specialized diabetes care. Edited by Dedov II, Shestakova MV ( $7^{\text {th }}$ edition). Diabetes mellitus. 2015;18(1S):1-112. doi: 10.14341/DM20151S1-112.

3. ISPAD Clinical Practice Consensus Guidelines 2014 Compendium. Pediatric Diabetes. 2014; 15(Suppl. 20): $1-290$.

4. King H., Aubert R.E., Herman W.H. Global burden of diabetes, 1995-2025: prevalence, numerical estimates, and projections. Diabetes Care, 1998; 21(9): 1414-1431.

5. Bykov I.M., GilmiYarova F.N., Domenyuk D.A., DMitrienko S.V., IVANyUta S.O., Budaychiev G. M-A. Evaluation of cariogenic situation in children with type 1 diabetes mellitus given the mineralizing potential of saliva and enamel resistance. Kubanskij nauchnyj medicinskij vestnik. 2018; 25(4): 22-36. (In Russ., English abstract). DOI: 10.25207 / 1608-62282018-25-4-22-36.

6. DOMENYUK D.A., ZELENSKY V.A., RZHEPAKOvsky I.V., Anfinogenova O.I., Pushkin S.V. Application of laboratory and x-ray gentral studies un early diagnostics of metabolic disturbances of bone tissue in children with autoimmune diabetes mellitus. Entomology and Applied Science Letters. 2018; 5(4): $1-12$.

7. Domenyuk D.A., Zelensky V.A., DMitrienko S.V., Anfinogenova O.I., Pushkin S.V. Peculiarities of phosphorine calcium exchange in the pathogenesis of dental caries in children with diabetes of the first type. Entomology and Applied Science Letters. 2018; 5(4): 49-64.

8. Domenyuk D.A., Konnov V.V., Pichugina E.N., Anfinogenova O.I., Goncharenko A.N., PushKIN S.V. Microcomputed tomography in qualitative and quantitative evaluation of dental enamel demineralization. Entomology and Applied Science Letters. 2018; 5(4): 72-83.

9. Bykov I.M., IVCHENKo L.G., DOMENYUK D.A., Kostyukova N.Y., Storozhuk A.P., ILIJEV D.M. 
Salivary the level of proinflammatory cytokines in children with autoimmune diabetes mellitus in different phases of compensation endocrinopathy. Kuban Scientific Medical Bulletin. 2017; 24(4): 39-48. (In Russ., English abstract). DOI: 10.25207/1608-62282017-24-4-39-48.

10. Davydov B.N., GilmiYarova F.N., Domenyuk D.A., IvChenko L.G. Clinical and diagnostic significance of the activity of matrix metalloproteinase and their tissue inhibitors in assessing the condition of periodontal tissues in children with type 1 diabetes mellitus. Part I. Children's dentistry and prevention. 2017; Vol. XVI; 4 (63): 14-19. (In Russ.).

11. Davydov B.N., Gilmiyarova F.N., Domenyuk D.A., IvChenko L.G. Clinical and diagnostic significance of the activity of matrix metalloproteinase and their tissue inhibitors in assessing the condition of periodontal tissues in children with type 1 diabetes mellitus. Part II. Children's dentistry and prevention. 2018; Vol. XVII; 1 (64): 37-46. (In Russ.).

12. Vedeshina E.G., Domenyuk D.A. Buccal epithelium reactivity indicators as markers of morphological and functional impairment in patients with dentoalveolar pathology. Kuban Scientific Medical Bulletin. 2016; (4): 23-32. (In Russ., English abstract). DOI:10.25207/1608-6228-2016-4-23-32.

13. Domenyuk D.A., Davydov B.N., Gilmiyarova F.N., IvChenko L.G., Vedeshina E.G. Diagnostic and prognostic value of crystalline structures of the oral fluid in children with anomalies of occlusion. Children's dentistry and prevention. 2017; Vol. XXI; 2(61): 9-16. (In Russ.).

14. IvChenko L.G., Domenyuk D.A. Diagnosis of immunometabolic disorders in children with type I diabetes mellitus. Kuban Scientific Medical Bulletin. 2017; 1(2): 73-82. (In Russ., English abstract). DOI:10.25207/1608-6228-2017-2-73-82.

15. Domenyuk D.A., Davydov B.N., Vedeshina E.G., DMitrienko S.V. Perfection of diagnostic methods of dentoalveolar anomalies by results of studying functional shifts in the system of oral homeostasis (Part I). The Dental Institute. 2016; 71(2): 74-77. (In Russ.).

16. Domenyuk D.A., Davydov B.N., Vedeshina E.G., DMitrienko S.V. Perfection of diagnostic methods of dentoalveolar anomalies by results of studying functional shifts in the system of oral homeostasis (Part II). The Dental Institute. 2016; 72(3): 58-60. (In Russ.).

17. Dmitrienko S.V., Davydov B.N., V.V. Shkarin, DoMENYUK D.A. Algorithm for determining the size of artificial teeth by the morphometric parameters of the face in people with full adentia. Dentistry. 2018; 97(6): 57-60.

18. Gilmiyarova F.N., Davydov B.N., Domenyuk D.A., IVCHENKo L.G. Influence of severity of type I diabetes mellitus in children on dental status and immunological, biochemical parameters of blood serum and oral fluid. Part I. Periodontology. 2017; Vol. XXII; 2 (83): 53-60. (In Russ.).
19. Gilmiyarova F.N., Davydov B.N., Domenyuk D.A., IVCHENKO L.G. Influence of severity of type I diabetes mellitus in children on dental status and immunological, biochemical parameters of blood serum and oral fluid. Part II. Periodontology. 2017; Vol. XXII; 3 (84): 36-41. (In Russ.).

20. DAVydov B.N., Domenyuk D.A., DMitrienKo S.V. Changes of the morphological state of tissue of the paradontal complex in the dynamics of orthodontic transfer of teeth (experimental study). Periodontology. 2018; Vol. XXIII; 1 (86): 69-78. (In Russ.).

21. KDIGO 2012 Clinical Practice Guideline forthe Evaluation and Management of Chronic Kidney Disease. Kidney Intern. Suppl. 2013; 3(1):1-150.

22. King H., Aubert R.E., Herman W.H. Global burden of diabetes, 1995-2025: prevalence, numerical estimates, and projections. Diabetes Care. 1998; 21(9): $1414-1431$.

23. Mc Carty D., Zimmet P. Diabetes. Global Estimates and Projections 1994 to 2010. International Diabetes Institute. 1994.

24. Takayanagi R., Inoguchi T., Ohnaka K. Clinical and experimental evidence for oxidative stress as an exacerbating factor of diabetes mellitus. J. Clin. Biochem. Nutr., 2010; 48(1): 72-77.

25. Dedov I.I., Shestakova M.V. Diabetes mellitus Type 1: reality and perspectives. Moscow: Meditsinskoe informatsionnoe agentstvo; 2016.

26. Dedov I.I., Balabolkin M.I., Mamaeva G.G. Diabetes mellitus: angiopathy and oxidative stress. Moscow, 2003. 86 p.

27. Zavodnyk I.B., Dremza I.K., Lapshina E.A. Diabetes mellitus: metabolic effects and oxidative stress. Journal of Membrane and Cell Biology. 2011; 2: 83-94.

28. Kolesnikova L.I., Osipova E.V., Grebenkina L.A. Oxidative stress in reproductive disorders of endocrine origin in women. Novosibirsk, Science, 2011. $116 \mathrm{p}$.

29. BYKov I.M., IVCHENKo L.G., DOMENYUK D.A., Kostyukova N.Y., StorozhuK A.P., ILIJEV D.M. Features of free radical oxidation and antioxidant protection in children with sugar diabetes of the first type. Kuban Scientific Medical Bulletin. 2017; (4): 27-38. (In Russ., English abstract). DOI: DOI:10.25207/1608-6228-2017-24-4-27-38.

30. Davydov B.N., Gilmiyarova F.N., Domenyuk D.A., IVCHENKo L.G. Optimization of diagnostics of type I diabetes in children according to the results of cytomorphological studies of buccal epithelium and processes of oxidative stress in the oral cavity. Children's dentistry and prevention. 2017; Vol. XVI; 3(62): 9-18. (In Russ.).

31. Davydov B.N., Domenyuk D.A., DMitrienko S.V. Changes of the morphological state of tissue of the paradontal complex in the dynamics of orthodontic transfer of teeth (experimental study). Periodontology. 2018; Vol. XXIII; 1 (86): 69-78. (In Russ.). 
32. Davydov B.N., Domenyuk D.A., Bykov I.M., IVChenko L.G., DMitrienko S.V. Modern possibilities of clinical, laboratory, X-ray studies in preclinical diagnosis and prediction of the risk of developing periodontal diseases in children with diabetes mellitus type one. Part I. Periodontology. 2018; Vol. XXIV; 3-24 (88): 4-11. (In Russ.). DOI: 10.25636/ PMP.1.2018.3.1

33. Valko M., Morris H., Cronin M.T. Metals, toxicity and oxidative stress. Curr. Med. Chem. 2005; 12(10): 1161-1208.

34. Davydov B.N., Gilmiyarova F.N., Domenyuk D.A., IVCHENKo L.G. Optimization of diagnostics of type I diabetes in children according to the results of cytomorphological studies of buccal epithelium and processes of oxidative stress in the oral cavity. Children's dentistry and prevention. 2017; Vol. XVI; 3(62): 9-18. (In Russ.).

35. Karslieva A.G., Domenyuk D.A., Zelensky V.A. Mixed saliva trace element composition in children with dentoalveolar anomalies through apparatusinvolved treatment. Archiv EuroMedica, 2014; 4(1): 29-35.

36. Dmitrienko S.V., Shkarin V.V., DaVydov B.N. Algorithm for determining the size of artificial teeth by the morphometric parameters of the face in people with complete adentia. Stomatologiya. 2018. 97 (6). P. 57-60. (In Russ.).

37. Bykov I.M., Basov A.A., Malyshko V.V., DzHImaK S.S., Fedosov S.R., Moiseev A.V. Dynamics of the pro-oxidant/antioxidant system parameters in wound discharge and plasma in experimental purulent wound during its technological liquid phase treatment. Bulletin of Experimental Biology and Medicine. 2017, 163 (2): 268-271.

38. Domenyuk D.A., Porfyriadis M.P., BudayCHIEV G. M-A. Contemporary methodological approaches to diagnosing bone tissue disturbances in children with type i diabetes. Archiv EuroMedica, 2018; 8(2): 71-81.

39. Gavrilova O.A., Domenyuk D.A. Specific features of oral cavity microbiocenosis in children using non-removable orthodontic appliances. Archiv EuroMedica, 2018; 8(2): 91-92.

40. Gavrilova O.A., Domenyuk D.A. Microbiological verification for the use of thermoplastics in prosthetic treatment of dentition issues in children. Archiv EuroMedica, 2018; 8(2): 88-90. 